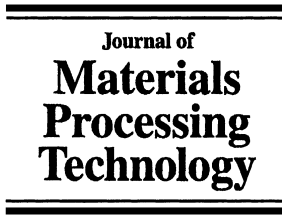

\title{
The influence of ductile interlayers on the mechanical performance of tungsten nitride coatings
}

\author{
M.T. Vieira ${ }^{*}$, A.S. Ramos \\ ICEMS - Faculdade de Ciências e Tecnologia da Universidade de Coimbra, 3030 Coimbra, Portugal
}

\begin{abstract}
Multilayers between ceramic materials and metallic materials can combine the high hardness and wear resistance of the ceramic layers with the toughness and mechanical strength of the metallic layers. This work is aimed at the production and characterisation of tungsten nitride/titanium (or nickel) multilayers. The coatings, deposited by magnetron sputtering, are characterised with respect to their structure, morphology and hardness. The adhesion to the substrate is evaluated by a scratch-test technique, as it constitutes a fundamental prerequisite.

The choice of titanium and nickel as interlayering materials was made in order to study the role of materials with elevated plasticity, but with different reactivities. Titanium tends to react chemically with other materials, namely, ferrous oxides and nitrides. Nickel has low chemical reactivity but has the same crystallographic structure as the $\mathrm{W}_{2} \mathrm{~N}$ phase. All of the multilayer coatings produced have a total thickness of $4 \mu \mathrm{m}$. The hardness of the multilayers, deposited with different ceramic/metal thickness ratios, decreases with the thickness of the ductile metallic layers. When the metal thickness is too high it causes the spalling of the coatings. The optimal medium critical load $(65 \mathrm{~N})$ is obtained with a ceramic/metal ratio equal to four. Even though the type of bond is different, the adhesion of the multilayers is not influenced by the substitution of titanium with nickel. The deposition process yields well-adhered and sufficiently hard multilayer coatings when compared with the ceramic single layers. (C) 1999 Elsevier Science S.A. All rights reserved.
\end{abstract}

Keywords: Multilayers; Ductile interlayers; Ultra-microhardness; Adhesion

\section{Introduction}

If the different layers are selected adequately, multilayer coatings can provide a way to enhance the performance of the system substrate/coating. Multilayer coatings have a wide range of applications being used as X-ray mirrors, monochromators, corrosion and erosion protections and diffusion barriers [1-5]. More recently, multilayer coatings have been used for mechanical application, namely to improve the wear resistance of coated components $[3,6,7]$. It seems that the wear resistance results from a specific favourable combination of hardness and toughness, which is difficult to obtain with a single coating [8]. The low toughness of hard ceramic coatings could be improved by bonding them with a ductile layer. Based on this principle the toughness of alumina coatings was increased by the incorporation of thin ductile nickel layers [8]. The introduc-

*Corresponding author. Tel: +351(0)39-7000745; fax: +351(0)397000701

E-mail address: teresa.vieira@mail.dem.uc.pt (M.T. Vieira) tion of the metal films aims at: (i) enhancement of the plastic deformation of the coating; (ii) stress relaxation; (iii) reduction of porosity; and (iv) crack deflection.

However, particularly when dealing with multilayer coatings, the adhesion is very important because it is necessary to promote not only the bonding to the substrate but also that between all of the layers involved.

The objective of the present work is to study the effect of ductile metal interlayers on the adhesion and mechanical performance of hard ceramic coatings.

The ceramic material adopted was tungsten nitride, type $\mathrm{W}_{2} \mathrm{~N}$, which has an fcc structure, and the ductile metals were titanium (hcp) or nickel (fcc). This choice was made in order to enhance the influence of the chemical reactivity and structure on the adhesion and mechanical properties of the coated samples. Titanium has a high chemical reactivity in opposition to nickel, which has the same structure as $\mathrm{W}_{2} \mathrm{~N}$.

In the case of multilayer coatings, parameters such as the number of layers, the period thickness and the relationship between the thickness of the two deposited materials can 
play an important role. It is interesting also to study the type of interfaces: direct transition, graded transition; and ion etching. Graded transition avoids discontinuities, whilst intermediate etching could improve adhesion by pre-activating the preceding layer.

\section{Experimental}

\subsection{Deposition technique}

Tungsten nitride, titanium and nickel films, as well as multilayer coatings, were deposited by d.c. magnetron sputtering from $\mathrm{W}(150 \mathrm{~mm} \times 150 \mathrm{~mm} \times 5 \mathrm{~mm})$, Ti $(150 \mathrm{~mm}$ $\times 150 \mathrm{~mm} \times 6 \mathrm{~mm})$ and $\mathrm{Ni}(150 \mathrm{~mm} \times 150 \mathrm{~mm} \times 1 \mathrm{~mm})$ targets with power targets of $1,2.5$, and $1 \mathrm{~kW}$, respectively. Sputtering was performed in pure argon at $3 \times 10^{-1} \mathrm{~Pa}$ for the titanium and nickel metallic films. To obtain the tungsten nitride ceramic coatings, the depositions occurred in an argon/nitrogen mixture at a total pressure of $4 \times 10^{-1} \mathrm{~Pa}$ and with a $\mathrm{N}_{2} /$ Ar partial pressure ratio of $1.5 / 1$. The coatings were deposited on M2 (AISI) high-speed steel that had been treated and polished down to diamond paste. Before deposition, the sputtering chamber was evacuated by a turbomolecular pump down to a final pressure of $10^{-4} \mathrm{~Pa}$. Afterwards, the substrate surfaces were heated and ion cleaned by an ion gun. During the deposition, the substrates were at a negative bias of $70 \mathrm{~V}$. To deposit the ceramic/metal multilayers it was necessary to introduce a second cathode into the deposition chamber. The second cathode had an independent power source. The transition between the two different materials of the multilayers was made by connecting and disconnecting the power sources associated with each cathode. The time during which each power source was maintained connected was selected in order to obtain the desired ceramic/metal thickness ratio and a total thickness of $4 \mu \mathrm{m}$. Coatings with nitrogen graded transitions at the metal/ ceramic and ceramic/metal interfaces were obtained by increasing and decreasing the nitrogen gradually during $1 \mathrm{~min}$. This procedure is possible once the nitrogen is the reactive gas used to produce the ceramic layers, and its flow is easily controlled.

\subsection{Characterisation techniques}

Film properties were measured using the following equipment: (i) a perthometer C5D roughness tester for the determination of film thickness; (ii) a Cameca SX50 electron probe microanalysis (EPMA) apparatus to obtain the chemical composition of the coatings and X-ray maps with the distribution of different elements; (iii) a Philips PW 3040/00 $\mathrm{X}$-pert diffractometer with $\mathrm{Co} \mathrm{K}_{\alpha}$ radiation for the evaluation of film structure; (iv) a Jeol T330 scanning electron microscope (SEM) to examine the morphology of the films observed in cross-section after fracture of the coated samples; (v) a Fisherscope H100 ultra-microhardness tester equipped with a Vickers indentor for the determination of the hardness; and (vi) a CSem-revetest scratch-tester equipped with a diamond indentor to evaluate the adhesion to the substrate by determining the critical adhesive load.

\section{Results and discussion}

\subsection{Tungsten nitride single coatings}

First of all it was necessary to select the $\mathrm{N}_{2} / \mathrm{Ar}$ ratio to be utilised during the tungsten nitride sputtering depositions. It was known that the nitrogen content of the tungsten nitride coatings only increases until a critical $\mathrm{N}_{2} / \mathrm{Ar}$ ratio is attained [9]. This is the reason why the three $\mathrm{N}_{2} / \mathrm{Ar}$ relations presented in Table 1 were selected and the chemical composition and thickness of the coatings evaluated. The nitrogen content is directly proportional to the $\mathrm{N}_{2} / \mathrm{Ar}$ ratio. The higher nitrogen content obtained is similar to that found by Castanho [9], so it was chosen as the higher ratio for all of the future single ceramic and multilayer coatings. The chemical composition results show the presence of residual carbon (approx. 3 at\%) for all coatings. This carbon could have two origins: contamination of the tungsten target during its manufacturing (which was previously confirmed by EPMA) and/or contamination of the deposition chamber.

The ceramic coatings were also analysed by X-ray diffraction (Fig. 1). The structure identification indicated, as expected, the presence of the $\mathrm{W}_{2} \mathrm{~N}$ fcc phase [10]. Due to nitrogen excess it is more correct to indicate this as a $\mathrm{W}_{2} \mathrm{~N}_{1+x}$ phase, as proposed by Affolter et al. [11]. The nitrogen excess should be responsible for the deviations observed in the interplanar distances, because by occupying the interstitials the nitrogen promotes lattice distortions.

Table 1

Tungsten nitride single coatings

\begin{tabular}{lllll}
\hline $\mathrm{N}_{2} /$ Ar ratio & $\mathrm{W}(\%$ at $)$ & $\mathrm{N}(\%$ at $)$ & $\mathrm{C}(\%$ at $)$ & $\begin{array}{l}\text { Thickness } \\
(\mu \mathrm{m})\end{array}$ \\
\hline $1 / 1$ & 51.5 & 45.1 & 3.4 & 2.2 \\
$1.2 / 1$ & 50.5 & 46.4 & 3.1 & 2.0 \\
$1.5 / 1$ & 47.8 & 49.6 & 2.6 & 1.8 \\
\hline
\end{tabular}

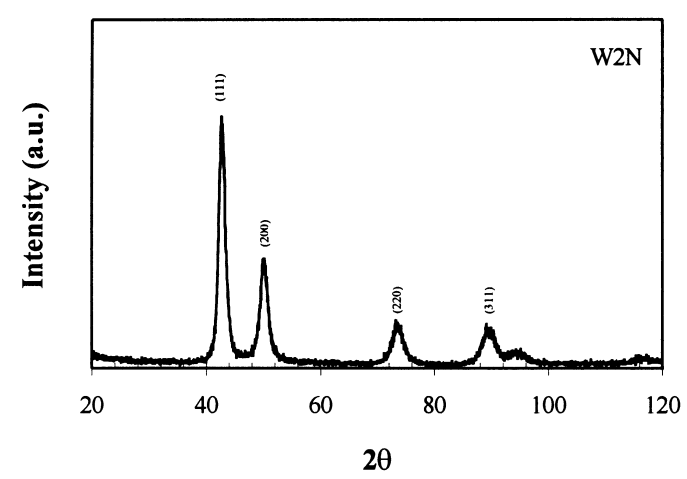

Fig. 1. X-ray diffraction results of tungsten nitride coatings. 
Table 2

Hardness and critical load of tungsten nitride coatings

\begin{tabular}{lll}
\hline Thickness $(\mu \mathrm{m})$ & Hardness $(\mathrm{GPa})$ & Lc2 $(\mathrm{N})$ \\
\hline 1.1 & 22.3 & $36.0 \pm 1.2$ \\
1.8 & 25.0 & $47.5 \pm 3.6$ \\
2.2 & 23.1 & $44.5 \pm 3.5$ \\
4.0 & 24.4 & $54.8 \pm 2.9$ \\
\hline
\end{tabular}

During the hardness tests substrate integration occurred, which had to be eliminated. To test the method adopted to eliminate the substrate influence on the determination of the hardness [12,13], coatings with different thickness were analysed. The hardness values and also the critical adhesive loads, Lc2, are presented in Table 2. The almost constant hardness values confirm the validity of the method used.

The tungsten nitride coatings failed by buckling (Fig. 2), which is a brittle film typical behaviour [14]. The critical adhesive load tended to increase with thickness, as shown in Table 2, which does not necessarily reveal better adhesion. The stresses that cause the failure of the coatings are transmitted through the coating across the interface, the attaining of this interface becoming more difficult as the coating thickness increases [15]. It should be noted that the adhesive failures occurred at the coating/substrate interface.

\subsection{Multilayer coatings}

Multilayer coatings were deposited in order to study the influence of the ceramic/metal thickness ratio (WN/M), graded transition and ion etching (Table 3), on the hardness and adhesion. The cross-sectional SEM images allow the identification of the different layers (Fig. 3), the dark zones corresponding to titanium or nickel whilst the bright zones correspond to tungsten nitride. According to Thornton's model, the morphology of the ceramic layers is type $\mathrm{T}$. The metal layers are featureless.

The hardness of the multilayers was evaluated using an ultra-microhardness tester. As a consequence of interlayer-

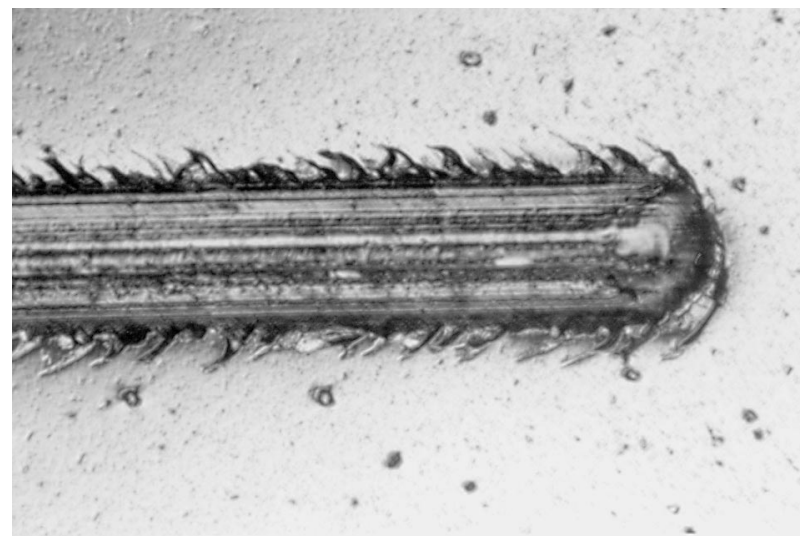

Fig. 2. Indentation channel of a tungsten nitride coating.
Table 3

Multilayer coatings

\begin{tabular}{lllll}
\hline Test & M & WN/M & $\begin{array}{l}\text { Graded } \\
\text { transition }\end{array}$ & Ion etching \\
\hline 1 & $\mathrm{Ti}$ & 2 & No & No \\
2 & $\mathrm{Ti}, \mathrm{Ni}$ & 4 & No & No \\
3 & $\mathrm{Ti}$ & 4 & Yes & No \\
4 & $\mathrm{Ti}$ & 4 & No & Yes \\
5 & $\mathrm{Ti}, \mathrm{Ni}$ & 8 & No & No \\
6 & $\mathrm{Ti}$ & 8 & Yes & No \\
7 & $\mathrm{Ti}$ & 8 & No & Yes \\
\hline
\end{tabular}

Table 4

Hardness of multilayer coatings

\begin{tabular}{ll}
\hline Multilayer & Hardness (GPa) \\
\hline $\mathrm{WN} / \mathrm{Ti}=8$ & 19.1 \\
$\mathrm{WN} / \mathrm{Ti}=4$ & 18.9 \\
$\mathrm{WN} / \mathrm{Ti}=2$ & 14.0 \\
$\mathrm{WN} / \mathrm{Ni}=8$ & 17.8 \\
$\mathrm{WN} / \mathrm{Ni}=4$ & 16.9 \\
\hline
\end{tabular}

ing soft metals inside a hard ceramic coating, the hardness decreases with the increase of the thickness of these layers (Table 4).

For a thickness ratio of 2, the hardness decrease is more pronounced, whilst for ratios of 4 and 8 the hardness value is almost equal. Multilayers with nickel as the intermediate material are softer than those with titanium, agreeing with the lower hardness value of nickel films.

The loading/unloading curves of: a multilayer coating with a thickness ratio of 4 ; a tungsten nitride $1.8 \mu \mathrm{m}$ thick coating; and a tungsten nitride $4.0 \mu \mathrm{m}$ thick coating are represented in Fig. 4. From this figure it is possible to conclude that the hardness behaviour of the multilayer is similar to that of a single tungsten nitride coating, since multilayer (total thickness $4 \mu \mathrm{m}$ ) and thicker ceramic coatings have identical loading/unloading curves.

Concerning the adhesion, there are different types of adhesive failures amongst the multilayer coatings, and even the same test can exhibit different behaviour when the coated samples are evaluated by the scratch-test (Fig. 5). There are also different interpretations of the scratch-test results. The present work followed a European pre-standard that is being reviewed [16]. Nevertheless, the most frequent adhesive failure is chipping characterised by coating removal on the channel sides. Spalling, only observed for a WN/Ti ratio equal to 2 , is characterised by the removal of the coating from inside of the indentation channel.

It is difficult to detect by optical microscopy if the substrate is exposed after indentation, but in Fig. 5(a) it seems that the substrate is visible on some of the removed zones. The SEM image of the end part of the indentation is clearer (Fig. 6). At the centre it is possible to see a removed zone (hole) and the different layers on its walls. The X-ray map of the iron along the indentation channel obtained with 

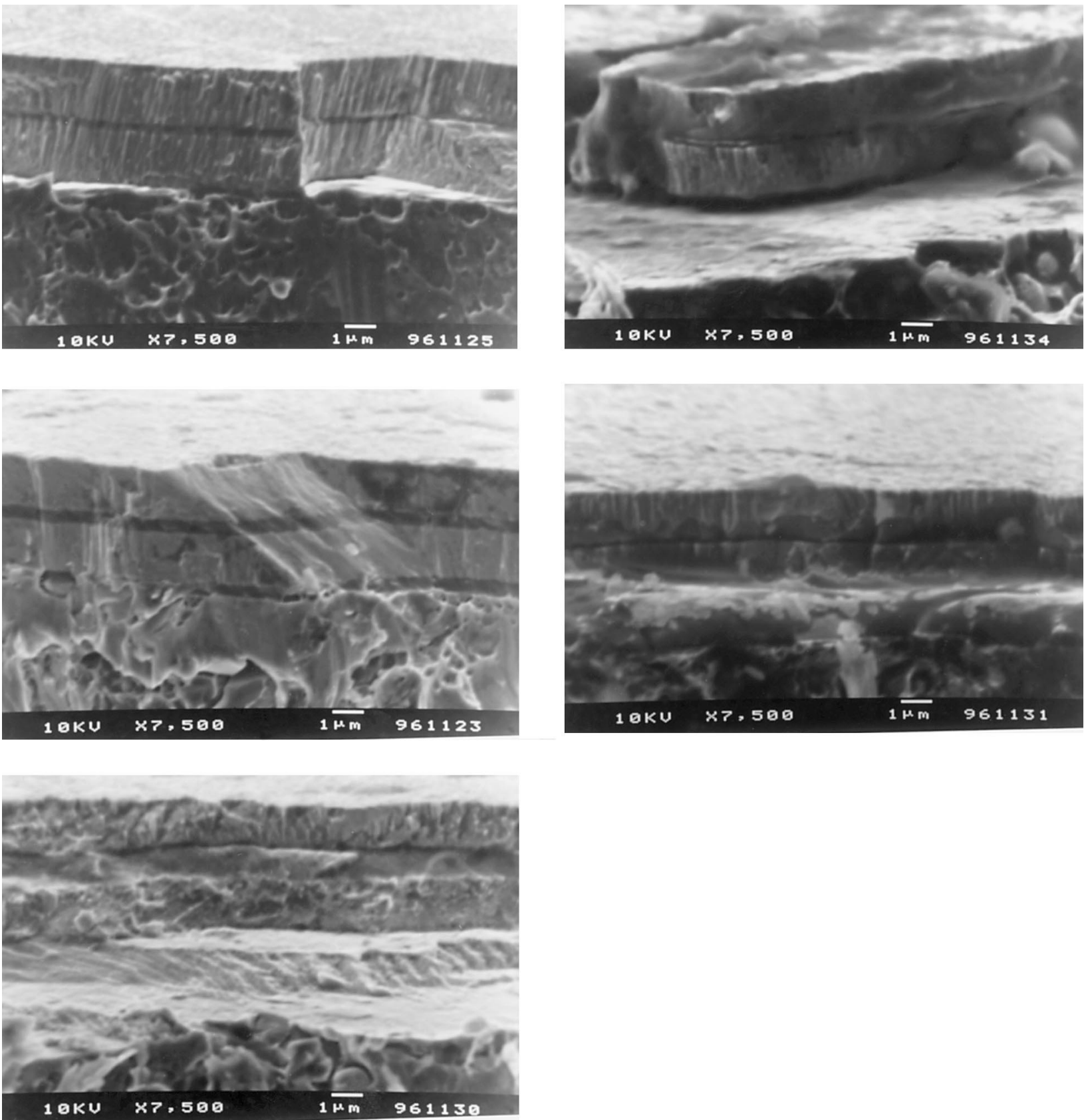

Fig. 3. Multilayer coatings SEM images: (a) WN/M=8; (b) WN/M=4; (c) WN/M=2.

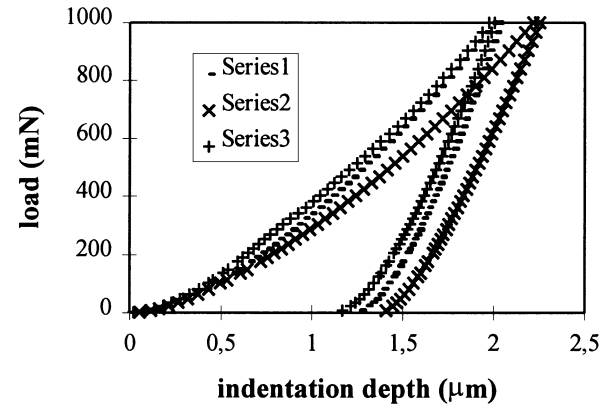

Fig. 4. Loading/unloading curves. (Series 1: multilayer coating ( $4 \mu \mathrm{m}$, $\mathrm{WN} / \mathrm{Ti}=4)$; Series 2 : tungsten nitride coating $(1.8 \mu \mathrm{m})$; Series 3 : tungsten nitride coating $(4.0 \mu \mathrm{m}))$.

the EPMA apparatus shows clearly the exposure of the steel substrate on the end of the scratch.

The adhesion of the coatings is related to the critical adhesive loads identified for the different tests that were made. Fig. 7 represents the critical medium loads and the associated standard deviation as a function of the test. Each test represents a combination of the three factors under study: thickness ratio; graded transition; and ion etching.

Comparing these results with those obtained with the single ceramic coatings, an increase of $10 \mathrm{~N}$ can be seen for the second test $(\mathrm{WN} / \mathrm{Ti}=4)$. TiN/Ti multilayers have a maximum Lc2 for an intermediate thickness of the Ti interlayers because it was sufficiently high to promote 


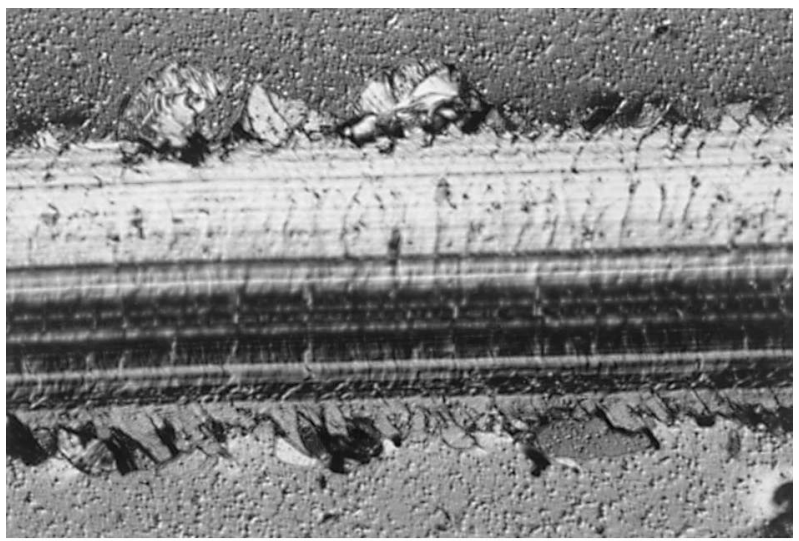

(a)
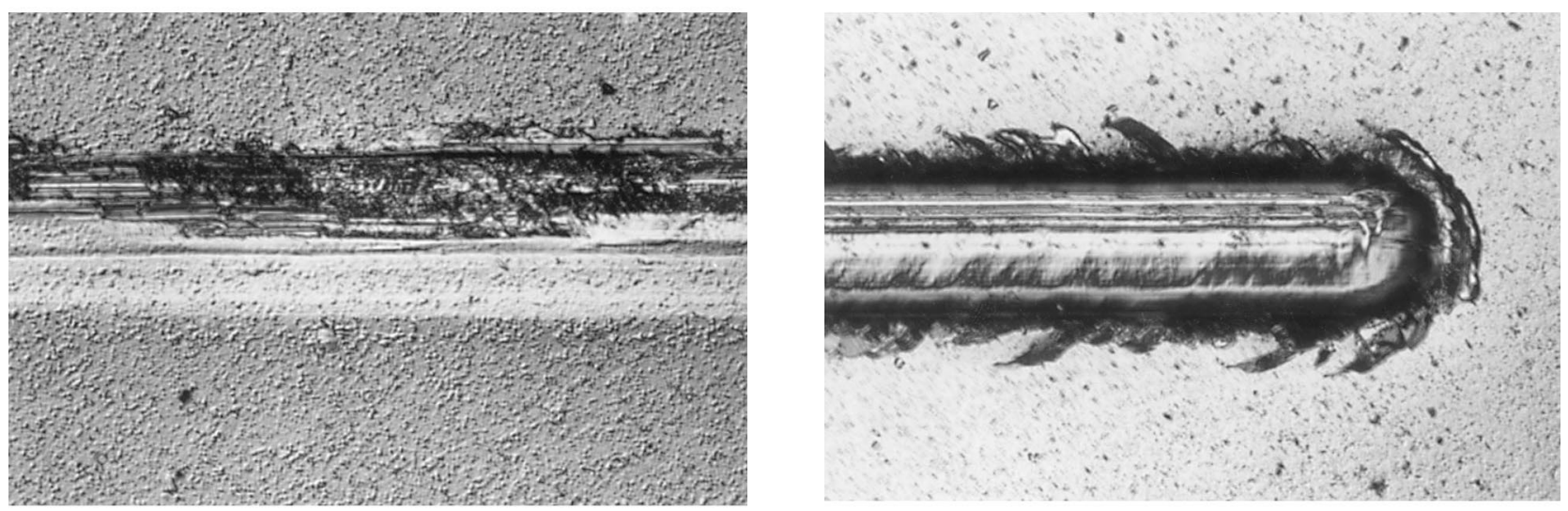

(b)

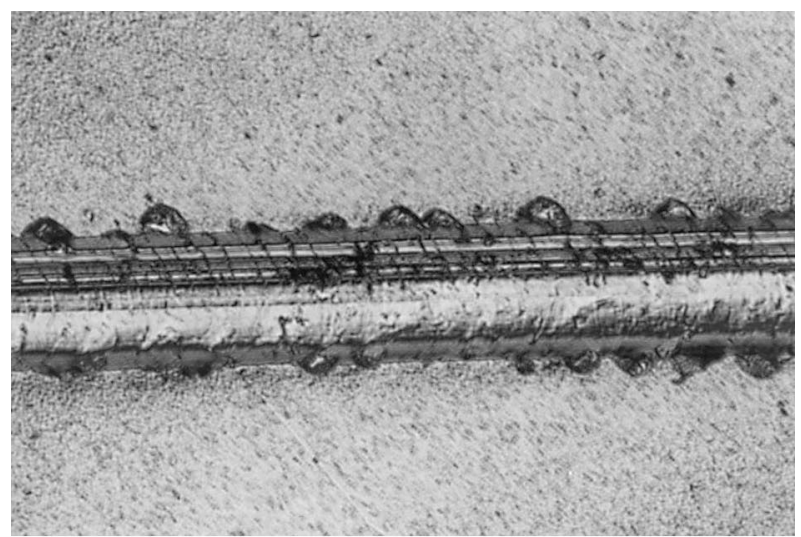

(c)

Fig. 5. Adhesive failures in multilayer coatings: (a) WN/Ti=4, chipping (200 X); (b) WN/Ti=2, spalling (200 X); (c) WN/Ni=8, chipping ( $100 \mathrm{X})$; (d) WN/ $\mathrm{Ni}=8$, small eruptions $(100 \mathrm{X})$.

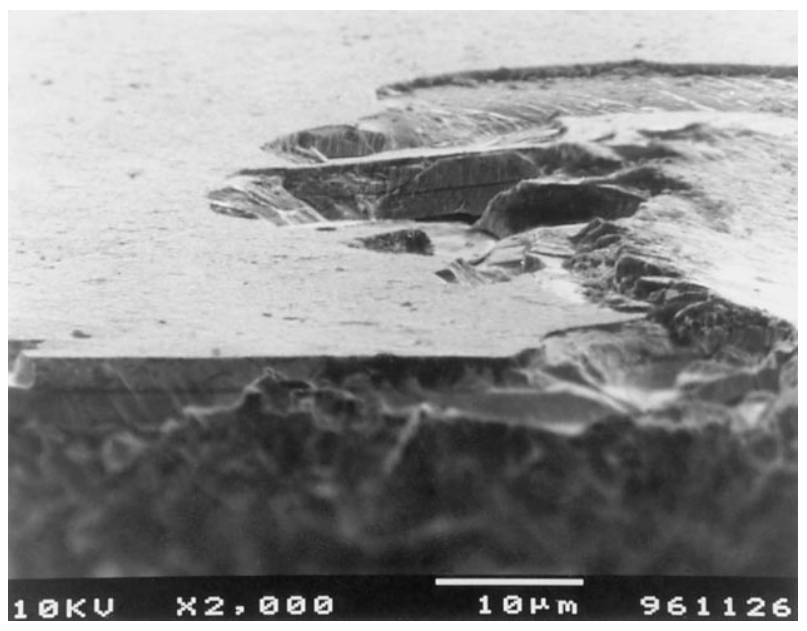

Fig. 6. End part of the indentation channel of a multilayer coating with $\mathrm{WN} / \mathrm{Ti}=8$.

energy absorption and not too high to lead to ductile failure in the titanium layers [17]. The thicker metal layers of the first test were responsible for the spalling of the samples that took place at low loads. The standard deviation related to the

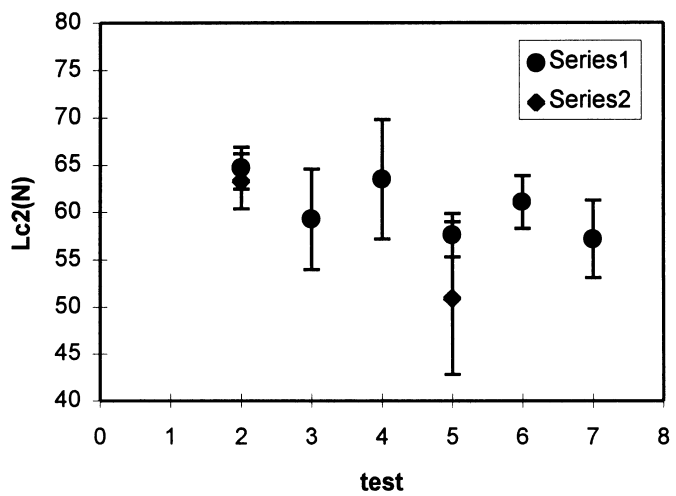

Fig. 7. Adhesive critical medium load and standard deviation. (Series 1: WN/Ti multilayers; Series 2: WN/Ni multilayers).

first test was too high and this was the reason why this test is not represented in Fig. 7. Considering the adhesion and hardness results, it can be concluded that the multilayer coatings with a ceramic/metal ratio equal to 2 have a great quantity of soft material compromising their behaviour and making them inadequate for mechanical applications. This phenomenon was also verified with $\mathrm{Al} / \mathrm{AlN}$ multilayers for tribological and corrosion protection, i.e. the thickness of the 
soft Al layers was too large to provide a good support for the AlN layers [18].

Introducing graded transitions and ion etching of the interfaces does not improve the adhesion and, foremost, as a result of a more complex experimental procedure, the standard deviation increases.

The results obtained with titanium and nickel did not reveal significant differences and, as was foreseen due to the high chemical reactivity of titanium, no improvement of adhesion was detected on the multilayers with titanium. Perhaps if a titanium alloy were used instead of pure titanium the adhesion would be better by forming $\mathrm{TiO}$ with the oxygen from the substrate surface. TiO is a metallic ceramic, thus promoting stronger bonding with metals, but this oxide is formed only for certain titanium activities [19], otherwise non-metallic Ti oxides are formed. Another way to improve the adhesion could be by increasing the amount of oxygen at the interface, proving the reactivity of the titanium.

\section{Conclusions}

Ceramic coatings, $\mathrm{W}_{2} \mathrm{~N}_{1+x}$, were produced by reactive sputtering, having a $24 \mathrm{GPa}$ hardness and an adhesive critical load of $55 \mathrm{~N}$. These characteristics make the $\mathrm{W}_{2} \mathrm{~N}_{1+x}$ appropriate for the coating of structural and mechanical parts. However, as with other hard coatings, these are fragile and easily propagate cracks, making them, in practice, inadequate. This could be overcome once it has been demonstrated that it is possible to introduce $\mathrm{Ti}$ and $\mathrm{Ni}$ ductile layers useful in the deflection of cracks that may be generated during the performance of the coated components. In fact, the hardness of the multilayer system is only slightly lower than that of the ceramic coatings. Further, the adhesion to the substrate is improved and a $65 \mathrm{~N}$ critical adhesive load was obtained for a tungsten nitride/titanium multilayer with a thickness ratio of 4 and of the same thickness as the ceramic coating mentioned above. Nevertheless, the introduction of metallic layers in the ceramic coatings resulting in a multilayer system is only beneficial if the ceramic/metal thickness ratio is not lower than 4 .

In conclusion, the structure and reactivity of metal interlayers do not seem to decrease the mechanical performance of multilayer coatings when compared to that of single ceramic coatings and they could be useful in crack deflection.

\section{References}

[1] Z. Jiang, J. Appl. Phy. 72 (1992) 931.

[2] A.F. Jankowski, M.A. Wall, J. Mat. Res. 9 (1994) 31.

[3] O. Hübler, A. Schröer, W. Ensinger, G.K. Wolf, W.H. Schreiner, I.J.R. Baumvol, Surf. Coat. Tech. 60 (1993) 561.

[4] E. Quesnel et al., Surf. Coat. Technol. 62 (1993) 474.

[5] P. Boher, P. Houdy, P. Kaïkati, L.J. Van Ijzendoorn, J. Vac. Sci. Technol. A8 (1990) 846.

[6] C. Subramanian, K.N. Straffoord, Wear 165 (1993) 85.

[7] H. Holleck, V. Schier, Surf. Coat. Technol. 76-77 (1995) 328.

[8] A.A. Minevich, Surf. Coat. Technol. 53 (1992) 161.

[9] J.M. Castanho, Influência do elemento intersticial no comportamento tribológico dos filmes do sistema W-N/C, University of Coimbra (1995).

[10] International Center for Diffraction Data, PA

[11] K. Affolter, H. Kattelus, M.-A. Nicolet, Proc. Symp. Mat. Res. Soc. 47 (1985) 167.

[12] A. Trindade, A. Cavaleiro, J.V. Fernandes, J. Test. Eval. 22 (1994) 365.

[13] J.V. Fernandes, Private Communication (1996).

[14] J. Ahn, K.L. Mittal, R.H. MacQueen, Hardness and adhesion of filmed structures as determined by scratch technique, American Society for Testing Materials, Miltall (1978).

[15] A.J. Perry, Thin Sol. Films 107 (1983) 167.

[16] European Pre-standard ENV 1071-3, Determination of adhesion by scratch-test (1994).

[17] K.J. Ma, A. Bloyce, T. Bell, Surf. Coat. Technol. 76-77 (1995) 297.

[18] R. Hübler, G.K. Wolf, W.H. Schreiner, I.J.R. Baumvol, Nuc. Inst. Met. Phy. Res. B80-B81 (1993) 1415.

[19] M.G. Nicholas, Joining of Ceramics, Chapman and Hall, London, 1990. 